\title{
L'Office provincial des recherches scientifiques et le développement de la science au Québec, 1937-1960
}

\section{Mike Almeida}

Volume 56, numéro 2, automne 2002

URI : https://id.erudit.org/iderudit/007316ar

DOI : https://doi.org/10.7202/007316ar

Aller au sommaire du numéro

Éditeur(s)

Institut d'histoire de l'Amérique française

ISSN

0035-2357 (imprimé)

1492-1383 (numérique)

Découvrir la revue

Citer cet article

Almeida, M. (2002). L’Office provincial des recherches scientifiques et le développement de la science au Québec, 1937-1960. Revue d'histoire de l'Amérique française, 56(2), 185-216. https://doi.org/10.7202/007316ar

\section{Résumé de l'article}

À sa création en 1937, l'Office provincial des recherches scientifiques avait pour mandat de coordonner la recherche effectuée dans les laboratoires de la province de Québec et de pourvoir à la formation de nouveaux chercheurs. En 1960, à l'époque des grands bouleversements institutionnels qui caractérisent la Révolution tranquille, il est intégré au Bureau des recherches économiques du ministère de l'Industrie et du Commerce et disparaît sous cette appellation. On a souvent affirmé que l'Office était un " échec relatif » dans le développement de la science au Québec. Une étude approfondie de l'organisme nous permet de nuancer ce jugement. En effet, nous avons découvert que l'agence gouvernementale finança, du moins en partie, pas moins de 173 mémoires de maîtrise et 56 thèses de doctorat, assurant ainsi un recrutement plus régulier d'étudiants des cycles supérieurs, là où s'acquiert le « métier " de chercheur et la connaissance des règles du jeu en vigueur dans chacune des disciplines scientifiques. De plus, l'Office s'est efforcé, dans sa première phase d'existence (1938-1948), d'orienter les recherches universitaires vers des applications pratiques à l'industrie, formant l'une des premières structures institutionnelles de liaison entre l'université et l'entreprise privée au Québec. Au cours des années 1950, l'Office est marqué par un changement de trajectoire. Le volet recherche de l'agence étatique prend alors la forme de ce que l'on pourrait appeler un « bureau gouvernemental de consultation technique et scientifique ». Ses activités de recherche se limitent donc à des analyses d'échantillons, au contrôle de la qualité de certains produits, à la solution de divers problèmes de fabrication, bref, à assister et à conseiller les entreprises qui en font la demande. Bien qu'il n'ait jamais eu l'ampleur ni les moyens du Conseil national de recherche du Canada (CNRC), l'Office a joué un rôle important dans la formation de la communauté scientifique québécoise.
Ce document est protégé par la loi sur le droit d'auteur. L’utilisation des services d’Érudit (y compris la reproduction) est assujettie à sa politique d'utilisation que vous pouvez consulter en ligne.

https://apropos.erudit.org/fr/usagers/politique-dutilisation/ 


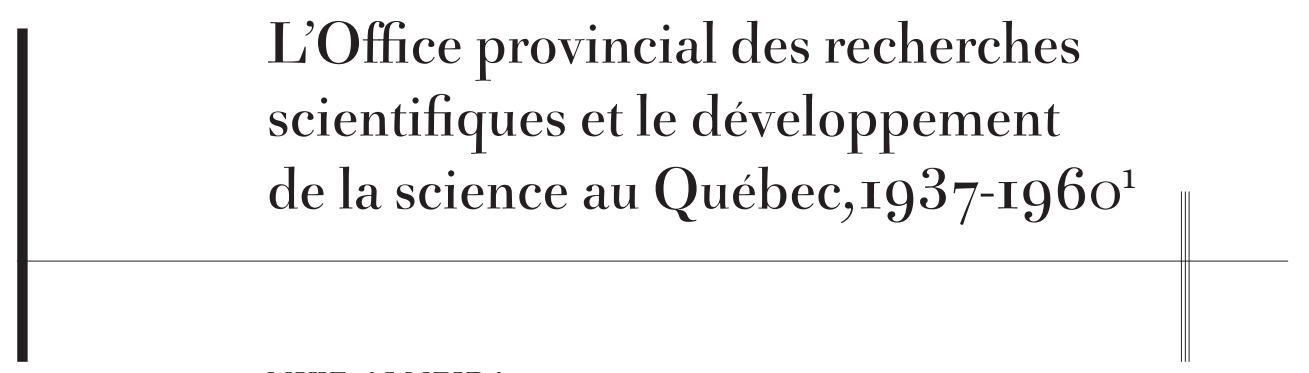

MIKE ALMEIDA

Centre interuniversitaire de recherche

sur la science et la technologie

Université du Québec à Montréal

RÉSUMÉ - À sa création en 1937, l'Office provincial des recherches scientifiques avait pour mandat de coordonner la recherche effectuée dans les laboratoires de la province de Québec et de pourvoir à la formation de nouveaux chercheurs. En 1960, à l'époque des grands bouleversements institutionnels qui caractérisent la Révolution tranquille, il est intégré au Bureau des recherches économiques du ministère de l'Industrie et du Commerce et disparaît sous cette appellation. On a souvent affirmé que l'Office était un «échec relatif» dans le développement de la science au Québec. Une étude approfondie de l'organisme nous permet de nuancer ce jugement. En effet, nous avons découvert que l'agence gouvernementale finança, du moins en partie, pas moins de 173 mémoires de maîtrise et 56 thèses de doctorat, assurant ainsi un recrutement plus régulier d'étudiants des cycles supérieurs, là où s'acquiert le «métier » de chercheur et la connaissance des règles du jeu en vigueur dans chacune des disciplines scientifiques. De plus, l'Office s'est efforcé, dans sa première phase d'existence (1938-1948), d'orienter les recherches universitaires vers des applications pratiques à l'industrie, formant l'une des premières structures institutionnelles de liaison entre l'université et l'entreprise privée au Québec. Au cours des années 1950, l'Office est marqué par un changement de trajectoire. Le volet recherche de l'agence étatique prend alors la forme de ce que l'on pourrait appeler un «bureau gouvernemental de consultation technique et scientifique ». Ses activités de recherche se limitent donc à des

1. L'auteur désire remercier Yves Gingras pour son encouragement indéfectible et l'attention précieuse qu'il a portée à la lecture des versions préliminaires de ce texte. Merci également à Jean-François Auger pour des conseils judicieux et à Colinda Julien pour la lecture et la relecture patiente du manuscrit. Ce travail aurait été impossible sans le concours généreux du Centre interuniversitaire de recherche sur la science et la technologie (CIRST). 
analyses d'échantillons, au contrôle de la qualité de certains produits, à la solution de divers problèmes de fabrication, bref, à assister et à conseiller les entreprises qui en font la demande. Bien qu'il n'ait jamais eu l'ampleur ni les moyens du Conseil national de recherche du Canada (CNRC), l'Office a joué un rôle important dans la formation de la communauté scientifique québécoise.

ABSTRACT - Created in 1937, the Scientific Research Bureau of the province of Quebec (1937-1960) was given the mandate to coordinate the research carried out in provincial laboratories and to assist the formation of young scientists by providing scholarships and grants. In the midst of the Quiet Revolution, it disappeared and was integrated to the Economic Research Bureau of the Trade and Commerce Department. For many historians, the Scientific Research Bureau did not really contribute to the growth of science in Quebec. Yet, a close analysis of the Bureau suggests otherwise. We discovered that it financed, at least in part, 173 master's degree and 56 Ph.D. Moreover, in its first phase of existence, the Bureau seeked to orient university-based research towards industrial application making it one of the first institutional links between the universities and the private sector in Quebec. At the turn of the 1950's, the Bureau changed its trajectory. The research it conducted thereafter was limited to sample analysis, quality control of products and counselling companies that seeked its aid. Even though the Bureau never had the financial resources of the National Research Council (NRC), it played a key role in the formation of the French-Canadian scientific community.

$\mathbf{F}$ ORMÉ EN I937 sous l'impulsion d'Esdras Minville, l'Office provincial des recherches scientifiques avait comme mandat de coordonner le travail de recherche effectué dans les laboratoires provinciaux, de faire porter ce travail sur les problèmes dont la solution était urgente pour l'économie du Québec et de pourvoir à la formation de nouveaux chercheurs par l'octroi de bourses d'études ${ }^{2}$. Jusqu'à maintenant, il était difficile d'en dire plus puisque l'Office n'avait pas encore fait l'objet d'une étude monographique. Raymond Duchesne est l'historien à y avoir consacré le plus d'attention avec huit pages dans son célèbre ouvrage La science et le pouvoir au Québec, paru en 1978. Pour Duchesne, l'Office était un "échec relatif» dans le développement de la science au Québec. D’abord, parce qu'il n'avait pas d'argent, son budget annuel de 15000 \$ étant maintenu intact de 1939 à 1961 «de telle sorte que, faute de pouvoir subventionner des projets d'envergure, l'Office [devait] renoncer très tôt à son rôle de coordonnateur des laboratoires existants ${ }^{3}$ ». Duchesne souligne ensuite que l'Office s'est contenté, tout au long de son histoire, d'accorder des bourses d'une valeur

2. Raymond Duchesne, La science et le pouvoir au Québec (1920-1965) (Québec, Éditeur officiel du Québec, 1978), 39.

3. Ibid., 41. 
moyenne de 500 \$ à des étudiants diplômés des facultés de sciences du Québec ${ }^{4}$. Il conclut finalement que les membres de l'organisme n'ont pas réussi à élaborer une approche valable au cours des années 1940 et 1950 et que, par conséquent, les politiques scientifiques du gouvernement provincial étaient aléatoires ${ }^{5}$.

Comme nous allons le voir, le laissez-faire caractéristique de l'époque et le sous-développement de l'appareil étatique ne laissaient aucune place à la planification économique et encore moins à une politique scientifique cohérente. En fait, la nature même de l'Office et les conditions dans lesquelles il a été créé interdisaient les notions de "planification nationale» et de "coordination interuniversitaire», notions qui n'apparaissent dans le vocabulaire des scientifiques québécois qu'au cours de la seconde moitié des années $1970^{6}$.

Quant aux ressources financières de l'Office, un coup d'œil rapide aux Comptes publics de la province de Québec suffit pour constater que si l'organisme avait effectivement un budget annuel de $15000 \$$ durant la Deuxième Guerre mondiale, il augmenta, par la suite, de manière constante pour atteindre $61700 \$$ en 1959, donc deux ans avant qu'il ne cesse d'exister (tableau 1$)^{7}$. Par ailleurs, le montant moyen des bourses d'études augmentait corrélativement à la hausse du budget de l'agence gouvernementale pour se fixer à $750 \$$ dès $1945^{8}$. Pour ce qui est du supposé dilettantisme des membres de l'Office qui auraient "préféré le silence à la réclame sauf pour demander une augmentation de leur indemnité ${ }^{\natural}$ ", là encore il appert qu'une lecture attentive des différents procès-verbaux des assemblées plénières de l'Office montre clairement des demandes répétées de crédits additionnels même pendant la guerre ${ }^{10}$.

4. Ibid.

5. Luc Chartrand, Raymond Duchesne et Yves Gingras, Histoire des sciences au Québec (Montréal, Boréal, 1987), 282.

6. Ibid., 429.

7. Comptes publics de la province de Québec, 1959.

8. Circulaire de l'Office provincial des recherches scientifiques pour l'année 1945, Fonds de la faculté des sciences (E0096/C4, 0018), Archives de l’Université de Montréal.

9. Raymond Duchesne, op. cit., 41.

10. À cet égard, on peut constater que le 18 décembre 1942 , lors de la $12^{\mathrm{e}}$ réunion plénière de l'Office, les membres présents ont décidé « d'orienter la recherche vers les problèmes intéressant l'effort de guerre et d'établir pour l'exercice financier 1943-1944 des prévisions budgétaires motivées en vue d'obtenir une augmentation du montant à distribuer en bourses». Procès-verbal de la $12^{\mathrm{e}}$ réunion plénière de l'Office provincial des recherches scientifiques, tenue aux bureaux du ministère du Commerce, à Québec, le vendredi 18 décembre 1942, Fonds de l'Institut de botanique (E118)A1, 1254, Archives de l’Université de Montréal. 
TA B LE A U 1

\section{La croissance des dépenses de l'Office provincial des recherches scientifiques 1949-1960}

\begin{tabular}{c|c|c|c}
\hline Année & \$ Courants & \$Constants & Taux de croissance (\%) \\
\hline $1949-1950$ & 17050 & 29396 & 100 \\
$1950-1951$ & 18425 & 30862 & 105 \\
$1951-1952$ & 20010 & 30318 & 103 \\
$1952-1953$ & 8525 & 12610 & 43 \\
$1953-1954$ & 31935 & 47664 & 162 \\
$1954-1955$ & 26910 & 39925 & 136 \\
$1955-1956$ & 29505 & 43711 & 149 \\
$1956-1957$ & 30500 & 44525 & 151 \\
$1957-1958$ & 32850 & 46463 & 158 \\
$1958-1959$ & 61700 & 84986 & 289 \\
$1959-1960$ & 50200 & 68392 & 233 \\
\hline
\end{tabular}

Les chiffres en dollars courants proviennent des Comptes publics de la province de Québec, 1949-1960. Les chiffres en dollars constants sont calculés d'après l'indice des prix à la consommation pour le Canada, 1913-1975. $197 \mid=100$. Le taux de croissance, établi sur la base des dollars constants, compare la valeur des dépenses annuelles à celle des dépenses de l'exercice 1949-1950.

Dans le présent article, nous proposons un premier relevé des activités de l'Office, tant au niveau des bourses d'études que de la recherche proprement dite. Pour l'instant, signalons que Le Devoir du 8 janvier 1948 annonçait que l'agence gouvernementale, après un peu plus de 10 ans d'existence, avait octroyé des bourses à plus de 160 chercheurs, dont une soixantaine dans le cadre d'une maitrise ès science, et environ vingt-cinq dans le but d'obtenir un doctorat. L'article en question rappelle que "plusieurs de ces jeunes chercheurs sont maintenant dans l'industrie ou au service des gouvernements fédéral et provincial ou encore occupent des chaires à l'Université Laval et à l'Université de Montréal ${ }^{11}$ ".

Sous cet angle, il nous apparaît clair que l'Office ne fut pas un échec, ni même un «échec relatif». Le dynamisme et la clairvoyance de ses membres, qui formaient une véritable génération de pionniers, ont permis de former des chercheurs et de contribuer à la création de conditions institutionnelles propices au développement de la recherche scientifique au Québec. Les

11. Le Devoir, jeudi le 8 janvier 1948. 
deux présidents de l'Office, Paul Riou et Jules Labarre, comptent parmi les premiers professeur-chercheurs à faire leur entrée dans le milieu de l'enseignement supérieur québécois. Ayant fait leurs études en Europe, ils ont importé des laboratoires étrangers l'habitus scientifique, «c'est-à-dire un ensemble de schèmes générateurs de pratique et de modes de perception et d'évaluation de ces pratiques ${ }^{12}$ ", condition nécessaire à l'émergence de la pratique scientifique au Québec. En subventionnant des travaux qui répondaient aux critères de cette pratique, les membres de l'Office ont contribué au développement de la communauté scientifique québécoise.

Quant à la recherche, l'Office a tenté, durant sa première phase d'existence (1938-1948), d'orienter les recherches universitaires vers des applications pratiques à l'industrie, formant l'une des premières structures institutionnelles de liaison entre l'université et l'entreprise privée au Québec. Nous verrons comment les années 1950 marquent un changement de trajectoire pour l'Office, qui se dote, en 1951, d'un comité des recherches appliquées, d'un laboratoire et d'un centre de documentation. Le volet recherche de l'agence étatique prend alors la forme de ce que l'on pourrait appeler un «bureau gouvernemental de consultation technique et scientifique». Au cours de sa dernière décennie d'existence, les activités scientifiques de l'Office se limitent donc à l'analyse d'échantillons, au contrôle de la qualité de certains produits, à la solution de divers problèmes de fabrication, bref, à assister et à conseiller les entreprises qui en font la demande.

Cet article vise à préciser le rôle et les attributs de l'État provincial dans ses liens avec l'université et l'industrie à travers une agence gouvernementale particulière, soit l'Office provincial des recherches scientifiques. Notre approche sera en quelque sorte une «biographie» institutionnelle, car retracer l'histoire d'une institution c'est suivre sa trajectoire au sein d'un espace social structuré en étant attentif aux bifurcations qui peuvent survenir en cours de route. Cette approche nous permet de concevoir notre objet de manière relationnelle et nous invite à présenter les caractéristiques des forces sociales qui entrent en interaction avec lui et qui influencent sa trajectoire. Bien sûr, comme l'a souligné Yves Gingras, "toute institution est composée d'individus, mais elle les transcende également en ce sens que, avec le temps, les individus passent alors que l'institution demeure ${ }^{13}$ ». C’est donc le récit d'une institution scientifique créée sous

12. Yves Gingras, Les origines de la recherche scientifique au Canada. Le cas des physiciens (Montréal, Boréal, 1991), 11.

13. Voir Yves Gingras, Pour l'avancement des sciences. Histoire de l'ACFAS, 1923-1993 (Montréal, Boréal, 1994), 10. 
Duplessis et emportée par la tourmente de la Révolution tranquille que l'auteur propose dans les pages qui suivent.

\section{LES DÉBUTS}

Pendant les années 1920, la communauté scientifique du Canada français prend forme avec la création de la faculté des sciences de l'Université de Montréal, l'École supérieure de chimie de l'Université Laval, l'ACFAS et plusieurs autres associations à vocation scientifique ${ }^{14}$. Ce mouvement d'institutionnalisation de la science prend abruptement fin avec la Grande Crise de 1929. L'urgence et la gravité des problèmes sociaux forcent la suspension, pendant plusieurs années, des projets envisagés par la communauté scientifique naissante et exacerbent les discussions sur la dépendance économique des Canadiens français. Sur cette question, le maître à penser est sans conteste le frère Marie-Victorin. Tout au long des années 1930, il affirme à plusieurs reprises que :

Pour conquérir la grande industrie, à ce moment de l'histoire où le capitalisme meurt intoxiqué par ses propres excès, il faut et il suffit qu'une élite d'hommes de science canadiens-français s'avère capable de faire face aux problèmes de l'industrie, qui sont avant tout des problèmes scientifiques, et seulement ensuite des problèmes d'organisation économique et des problèmes sociaux. Le jour où nous aurons cette élite scientifique, la reconquête par le paisible envahissement de la place ne sera qu'un jeu d'enfant ${ }^{15}$.

Marie-Victorin se faisait ainsi l'écho du mouvement technocratique alors en vogue aux États-Unis et qui revendiquait une gestion scientifique de la société ${ }^{16}$. Ce discours sur l'importance de la science allait prendre une portée nationale au cours de la campagne électorale de 1936.

En effet, au cours de cette campagne, la communauté scientifique appuie ouvertement l'Union nationale qui s'est engagée à moderniser le Québec par la nationalisation des ressources naturelles et la mise en vigueur de mesures sociales. Au pouvoir depuis plus de 15 ans, le Parti libéral de L.-A. Taschereau apparaît comme une formation corrompue et vieillie, incapable de faire face aux difficultés du temps. Le 17 août 1936, l'Union nationale fait élire 76 députés et remporte une victoire éclatante. Le soutien sans réserve que la communauté scientifique et intellectuelle avait

14. Luc Chartrand et al., op. cit., 273.

15. Marie-Victorin, Science, culture et nation, textes choisis et présentés par Yves Gingras (Montréal, Boréal, 1996), 161.

16. Ibid. 
témoigné à l'égard du nouveau gouvernement se traduit rapidement par un rapprochement des scientifiques avec certains éléments du champ politique. En 1937, l'accession de Georges Maheux, ingénieur forestier et président de l'ACFAS en 1935, au poste de directeur du Service de protection des plantes, et la nomination de Jean Bruchési, conseiller de l'ACFAS, comme sous-secrétaire de la province, sont autant d'exemples qui confirment ce rapprochement ${ }^{17}$. La nomination de plusieurs scientifiques à des postes clés du gouvernement permettait d'augmenter leur influence sur l'ensemble de la société et la plupart allait en profiter pour relancer l'institutionnalisation de la science amorcée au cours des années 1920. Le succès de cette relance tenait, dans une large mesure, au fait que les scientifiques parvenus au pouvoir partageaient et collaboraient volontiers au programme socio-économique du nouveau gouvernement unioniste ${ }^{18}$.

Ainsi, Esdras Minville, directeur de L'Actualité économique et professeur à l'École des hautes études commerciales, acceptait le poste de conseiller technique auprès du ministre de l'Industrie et du Commerce, l'honorable Joseph Bilodeau ${ }^{19}$. Aidé par le sous-ministre Louis Coderre, également des HEC, Minville avait pour tâche d'organiser le ministère qui avait été créé au courant de l'année 1936. L’une des premières initiatives de ce ministère fut de promulguer la loi pour établir l'inventaire des ressources naturelles de la province; à cette fin, le ministère était autorisé à dépenser 100000 \$. En outre, les dispositions de la loi permettaient la réorganisation du service de statistiques de la province ainsi que la création d'un service de renseignements commerciaux, d'un service de recherches économiques et, finalement, d'un service de recherches scientifiques ${ }^{20}$.

17. Luc Chartrand et al., op. cit., 277.

18. Rappelons que le gouvernement de Maurice Duplessis allait abandonner ses promesses de nationalisations pour se concentrer sur l'exploitation des ressources naturelles par des capitaux étrangers. Pour plus de détails, voir Kenneth McRoberts et Dale Posgate, Développement et modernisation du Québec (Montréal, Boréal, 1983), 79-114.

19. Il faut dire que Minville avait même été sollicité par Maurice Duplessis pour être candidat aux élections avec la perspective d'un portefeuille si le parti était élu. Puis, après les élections, le premier ministre lui avait offert le poste de sous-ministre à l'Industrie et au Commerce, mais sans succès. Il acceptait finalement la charge de conseiller technique. Selon la $16^{\mathrm{e}}$ causerie radiophonique prononcée par Minville sur les ondes de CKAC au printemps de 1957, Fonds Esdras Minville (P035), Archives des HEC. Voir également l'article de François-Albert Angers et de Roland Parenteau, «Statistiques manufacturières du Québec, 1665-1948», L'Actualité économique (janvier 1966) : 3.

20. Loi ordonnant l'inventaire des ressources naturelles de la province de Québec. Une copie du texte de loi se trouve au dossier «Inventaire des ressources naturelles» du Fonds Esdras Minville (P035), Archives des HEC. 
Dans un communiqué de presse du 7 juin 1937, le ministre Bilodeau justifie ainsi la mise en vigueur de la nouvelle loi : «le développement de nos industries est lié à l'exploitation de toutes nos richesses naturelles [...] et non seulement voulons-nous maintenir les industries actuelles, nous entendons encore favoriser l'établissement de nouvelles usines et fabriques. »Se faisant rassurant, le ministre rappelle qu'il n'entend point substituer l'État aux citoyens, ni s'ingérer dans l'entreprise privée; il s'agit plutôt de fournir à la collectivité les renseignements et la documentation dont elle a besoin pour prospérer. À ce propos, il annonçait fièrement que son ministère allait bientôt être en mesure, par l'entremise de l'Office des renseignements commerciaux, du Bureau des statistiques et de l'Office des recherches économiques, d'offrir aux industriels, aux commerçants et aux financiers des précisions sur les marchés intérieurs et extérieurs. Du reste, l'Office des recherches scientifiques, assuré du bon concours des divers laboratoires de la province, pourrait résoudre les problèmes qu'une telle entreprise ne manquerait pas de poser ${ }^{21}$.

La mise sur pied de ces divers organismes, dont l'Office des recherches scientifiques, était proposée par Minville et Coderre dans un document interne adressé au ministre et intitulé "Arguments en faveur d'un Office de recherches et de renseignements ${ }^{22}$ ». Partant toujours des mêmes prémisses (l'État ne saurait agir efficacement sans la connaissance précise des ressources du territoire; la meilleure façon pour l'État d'aider l'initiative privée consiste à lui fournir non pas des fonds, mais des services; etc.), les différents organismes étaient inspirés de ceux créés en Angleterre, pays qui, selon les auteurs du document, s'était rapidement relevé de la crise économique grâce à cette politique d'assistance technique à l'industrie et au commerce ${ }^{23}$. Minville et Coderre estimaient par ailleurs que la création de ces différents services offrirait un débouché aux diplômés universitaires en facilitant l'accès à des carrières scientifiques. Le mémorandum soulignait en outre que le Québec avait des hommes de science, mais que ces derniers s'étaient, jusque-là, consacrés essentiellement à l'enseignement. Afin de corriger la situation, les auteurs voyaient dans l'édification

21. Communiqué de presse daté du 7 juin 1937 émis par l'honorable Joseph Bilodeau, ministre de l'Industrie, du Commerce et des Affaires municipales, figurant dans le Fonds Esdras Minville (P035), Archives des HEC.

22. Ce document est contenu dans la série «Inventaire des ressources naturelles» du Fonds Esdras Minville (P035), Archives des HEC.

23. Les auteurs renvoient à un article du Bulletin commercial de février 1936 reproduit dans l'Actualité économique, 11,2 (novembre 1935-mars 1936) : 476-482. 
d'un office des recherches scientifiques le moyen de stimuler la recherche et de la faire porter sur des problèmes locaux : pêcheries, agriculture, forêts, mines, industrie manufacturière, etc. ${ }^{24}$.

C'est dans cette optique que Minville convoque, le 4 décembre 1937, plusieurs savants parmi les plus en vue - Marie-Victorin, Adhémar Mailhiot, directeur de Polytechnique, Alexandre Vachon, directeur de l'École supérieure de chimie de l'Université Laval — afin de les consulter sur la structure et l'orientation prévues pour le futur organisme. Après quelques délibérations, l'assemblée en vint à la conclusion «que l'office proposé pourrait rendre les plus grands services» :

1. En coordonnant le travail de recherche des laboratoires existants qui, aujourd'hui, procède un peu selon la fantaisie des chercheurs (il est bien entendu que nous ne songeons pas, à l'instar du Service de recherche à Ottawa, à bâtir des laboratoires nouveaux). 2. En faisant porter ce travail sur des problèmes de chez nous, élucidant une foule de questions dont la solution est des plus urgentes. 3. En aidant d'une façon indirecte les hommes de science actuels et surtout les jeunes que la carrière scientifique pourrait tenter ${ }^{25}$.

Esdras Minville, qui dirigeait également l'Office des recherches économiques chargé de l'inventaire des ressources naturelles, fut nommé président, Georges Maheux, entomologiste provincial, vice-président, et Fortunat Fortier, diplômé des HEC, secrétaire. On priait ensuite «le ministre de nommer membre de l'Office $[\ldots]$ les scientifiques présents à la réunion plus Henri Roy, du ministère des Terres et Forêts, A.-O. Dufresne, du Service des mines, le docteur Georges Préfontaine, professeur à l'Université de Montréal et, pour faire bonne mesure, un représentant de McGill» qui restait à déterminer ${ }^{26}$. Acquiesçant à toutes ces

24. Arguments en faveur d'un Office de Recherches et de Renseignements, 2, non daté, contenu dans la série «Inventaire des ressources naturelles» du Fonds Esdras Minville (P035), Archives des HEC.

25. Luc Chartrand et al., op. cit., 281.

26. Ibid. Voici la liste complète des membres fondateurs de l'Office provincial des recherches scientifiques telle que rapportée par L'Action universitaire, 4,5 (janvier 1938) : 87 : Esdras Minville, professeur aux HEC, président, Georges Maheux, entomologiste provincial, vice-président, Fortunat Fortier, secrétaire. Les autres membres sont : Paul Riou, professeur aux HEC, Henri Roy, président de l'Association des ingénieurs forestiers, Georges Préfontaine, professeur à l'Institut de zoologie de l'Université de Montréal, L.-Z. Rousseau, Jacques Rousseau, professeur à l'Institut de botanique de l'Université de Montréal, Marie-Victorin, président de l'Institut de botanique de l'Université de Montréal, A.-O. Dufresne, directeur du Service des mines du gouvernement du Québec, l'abbé Vachon, directeur de l'École de chimie de l'Université Laval, et Adhémar Mailhot, directeur de l'École polytechnique de Montréal. 
demandes, le ministre Bilodeau inaugurait officiellement la création de l'Office des recherches scientifiques de la province de Québec lors d'une conférence de presse tenue le 22 décembre 1937 dans la Vieille Capitale ${ }^{27}$.

Longtemps après la tenue de cette réunion, Esdras Minville rappelait, dans le cadre d'une causerie radiophonique, que son rôle dans la création de l'Office s'était limité à en faire accepter l'idée et à remplir la fonction, assez vague, de président durant la période d'organisation ${ }^{28}$. L'assertion implicite de Raymond Duchesne selon laquelle Minville aurait «cédé» la présidence de l'Office dans un mouvement général de "défection" des membres de l'organisme est peu probable ${ }^{29}$. En fait, Minville entretenait de grandes espérances envers l'Office, comme il le souligna lors de cette même émission radio : "Nous espérions, qu’à plus ou moins longue échéance, l'Office élargirait son champ d'initiative et entreprendrait luimême des recherches appliquées, en relation avec les travaux de l'Office des recherches économiques, notamment sur l'utilisation des ressources exploitées et exploitables des diverses régions de la province ${ }^{30}$. " L’idée de voir un jour l'Office doté de ses propres laboratoires, à l'instar du CNR, avait donc été caressée dès le début, malgré la modestie apparente des membres fondateurs.

De toute façon, Minville, licencié en sciences commerciales, avouait luimême ne pas posséder les connaissances ni les aptitudes nécessaires pour diriger un tel organisme. Il s'agissait donc pour lui d'assurer l'existence de l'institution par une législation et des crédits appropriés, pour ensuite passer le flambeau à un homme de science aguerri, capable de coordonner et d'orienter le travail des chercheurs ${ }^{31}$. Appelé au poste de directeur de l'École des hautes études commerciales en 1938, il démissionne de la présidence de l'Office et recommande au ministre la nomination de son collègue Paul Riou, professeur de chimie à l'École des HEC.

Né en 1890 à Trois-Pistoles, Riou fait partie de la première promotion de diplômés de l’École des HEC où il obtient, en 1913, la licence en sciences commerciales. Préparateur au laboratoire de chimie de l'institution puis chargé de cours, il décide, en 1920, de s'embarquer pour Paris et s’inscrit à

27. Le Devoir, mercredi le 22 décembre 1937.

28. Selon la $16^{\text {e }}$ causerie radiophonique prononcée par Minville sur les ondes de CKAC au printemps de 1957, Fonds Esdras Minville (P035), Archives des HEC.

29. Raymond Duchesne, op. cit., 40.

30. Selon la $16^{\mathrm{e}}$ causerie radiophonique prononcée par Minville sur les ondes de CKAC au printemps 1957, Fonds Esdras Minville (P035), Archives des HEC.

31. Ibid. 
la Sorbonne où il se livre à des recherches sur les vitesses de réaction en milieu hétérogène sous la direction du célèbre chimiste Henry Le Châtelier ${ }^{32}$. En 1922, il obtient sa licence ès science et l'Académie des sciences de Paris lui décerne une bourse afin qu'il puisse poursuivre ses travaux jusqu'au doctorat. Un an plus tard, il devenait l'un des premiers titulaires canadiens d'un doctorat émis par l'État français ${ }^{33}$. Boursier du CNR dès 1924 pour ses travaux sur la forme cristalline du bicarbonate de soude, Riou est surtout connu pour son célèbre manuel de chimie, rédigé avec l'aide de son collègue des HEC, Gérard Delorme, et réédité plusieurs fois à l'intention des étudiants "des collèges classiques et des grandes écoles ${ }^{34}$ ». Le spécialiste en cinétique chimique a rédigé plusieurs articles, seul ou en collaboration, et fait partie de cette première génération de chercheurs francophones qui ont importé des laboratoires européens le savoir-faire que requiert la pratique de la recherche scientifique. Riou était donc le candidat idéal, d'autant plus qu'il avait été formé, au cours de son séjour à la Sorbonne, par des scientifiques qui tentaient de faire tomber les barrières traditionnelles entre la science fondamentale et la science appliquée ${ }^{35}$. Il acceptait finalement l'offre du ministre et allait occuper la présidence jusqu’en décembre 1947.

Bien que Marie-Victorin ait salué la création de l'Office parce qu'il détruisait «les cloisons, autrefois étanches, entre la science, l'administration et la politique ${ }^{36}$ ", il serait inadéquat d'y voir un premier pas vers la planification économique ou pire, un accroissement de l'interventionnisme étatique. En effet, nous avons montré comment les prémisses sur lesquelles

32. Henry Le Châtelier (1850-1936) est surtout connu pour ses pyromètres thermo-électrique (1886) et optique (1892) qui permirent de créer la chimie de précision aux températures élevées. Fondateur de la Revue de Métallurgie (1904), membre de l'Académie des sciences de Paris (1907), grand promoteur et ami du célèbre ingénieur américain Frederick W. Taylor, Le Châtelier a consacré toute sa vie à forger ce qu'il appelait la science industrielle : «mon but est de combattre le sentiment aujourd'hui très général en France que la science doit rejeter loin d'elle toute préoccupation des applications pratiques, qu'elle doit s'isoler de l'industrie comme d'une promiscuité compromettante [...]. La fusion de la théorie et de la pratique doit aujourd'hui être la préoccupation dominante de tous ceux qui s'intéressent, tant au progrès de la science pure qu'à celui de l'industrie.» Cité dans Georges Charpy, «Henry Le Châtelier et la métallurgie», Revue de métallurgie (janvier 1937) : 19. Voir également les articles de Léon Guillet et Albert Portevin dans le même numéro de la Revue de métallurgie, 1-53. Henry Le Châtelier a explicité son programme dans Science et Industrie (Paris, Flammarion, 1925), $283 \mathrm{p}$.

33. Les nouvelles de l'École des hautes études commerciales, 3,9-10 (décembre 1929-janvier 1930) : 1-2.

34. Paul Riou et Gérard Delorme, Traité de chimie (Montréal, Beauchemin, 1947), 615 p.

35. Voir Terry Shinn, "Change or Mutation? Reflections on the Foundations of Contemporary Science", Social Science Information/Information sur les sciences sociales, 38,1 (1999: 149-176). Voir également Henry Le Châtelier, Science et industrie, op. cit.

36. Marie-Victorin, op. cit., 159. 
reposait l'Office lui attribuaient un rôle supplétif visant essentiellement à épauler l'initiative privée. Agissant en accord strict avec les principes du libéralisme économique, l'Office ne pouvait remplir son mandat tant attendu de coordonnateur des recherches effectuées dans les laboratoires provinciaux. C'est que règle générale, les conseils scientifiques, chargés de coordonner de larges équipes de chercheurs, sont apparus dans le sillage de la Première Guerre mondiale qui nécessitait, de la part des États belligérants, une prise en charge de larges pans de l'économie et la coordination de vastes projets de recherches industrielles et militaires ${ }^{37}$.

Or, dans le cas du Québec, l'Office apparaît en réponse à la crise économique, mais le gouvernement ne modifie aucunement ses conceptions et ses pratiques concernant le rôle de l'État, de sorte que le mandat de coordination des recherches, qui avait été confié à l'Office par ses fondateurs, était, pour ainsi dire, inconséquent. En effet, pour qui l'Office aurait-il pu effectuer ce travail de coordination si ce n'est pour l'État? Rappelons que les grandes entreprises qui exploitaient les richesses naturelles de la province appartenaient à des capitaux américains ou canadiens-anglais et que ces entreprises étaient munies de leurs propres laboratoires de recherche et de développement, basés pour la plupart aux États-Unis. Il semble que les scientifiques canadiens-français, qui ne réussissaient pas encore à percer sur la scène fédérale, aient vu dans la création d'un Office provincial, calqué sur le modèle du CNR, le moyen de mettre à leur disposition des ressources matérielles et humaines non négligeables. Quand on pense qu'en 1933, seulement $3 \%$ des ingénieurs et des scientifiques à l'emploi du gouvernement fédéral étaient des francophones, on ne peut guère s'étonner que la jeune communauté scientifique canadienne-française se soit tournée vers le gouvernement provincial pour obtenir du financement ${ }^{38}$.

37. Sur les origines du Conseil de recherche britannique, voir R. M. MacLeod et E. Kay Andrews, "The Origins of the DSIR : Reflections on Ideas and Men, 1915-1916", Public Administration, 48 (1970) : 23-48. Pour l'exemple canadien voir Wilfrid Eggleston, National Research in Canada. The NRC, 1916-1966 (Toronto, Clark Irwin, 1978), 470 p.; et Yves Gingras, Les origines..., op. cit. Pour l'exemple de l'Australie voir C. B. Schedvin, Shaping Science and Industry : A History of Australia's Council for Scientific and Industrial Research, 1926-1949 (Sydney, Allen \& Unwin, 1987). Pour le cas indien voir V. V. Krishna, "Organisation of Industrial Research : The Early History of CSIR", Technology and the Raj : Western Technology and Technical Transfers to India, 1700-1947 (New Delhi and Thousand Oaks (CA), Sage, 1995), 289-323.

38. Raymond Duchesne, op. cit., 21. 


\section{LES BOURSES D'ÉTUDES}

À compter de l'automne 1938, au début de chaque trimestre universitaire, la circulaire d'offre de bourses de l'Office était affichée dans tous les départements et facultés de sciences des universités du Québec. La circulaire annonçait des bourses d'études au montant de $500 \$$ pour la première année, $600 \$$ pour la deuxième et $700 \$$ pour les années suivantes ${ }^{39}$. Les montants en bourses de l'Office étaient donc comparables aux bursaries du CNR d'une valeur de $500 \$$ par année et au studentships d'une valeur de $600 \$$ la première année et de $750 \$$ la seconde. Seuls les fellowships, permettant aux étudiants d'obtenir un doctorat, représentaient un montant nettement supérieur aux bourses d'études de l'Office avec une valeur de $1000 \$$ la première année et $1200 \$$ la seconde ${ }^{40}$.

Les candidats intéressés à obtenir une bourse d'étude de l'Office devaient résider dans la province depuis au moins un an et témoigner d'une formation scientifique suffisante : licencié ès science, génie ou diplôme équivalent. En outre, le bénéficiaire devait consacrer tout son temps à la recherche durant dix mois consécutifs, à moins d'une entente avec l'Office. Il s'engageait également à obtenir son admission dans un laboratoire reconnu par l'organisme et à fournir un rapport mensuel sur l'état de ses travaux, rapport qui devait être contresigné par le directeur de la recherche. Par ailleurs, le titulaire d'une bourse de l'Office ne pouvait être en même temps bénéficiaire d'une bourse ou d'une subvention d'un autre organisme de recherche, il ne devait rien divulguer de ses travaux sans l'autorisation de l'Office et si une découverte donnait lieu à un brevet, le chercheur pouvait en bénéficier dans une proportion définie entre lui et l'agence gouvernementale $^{41}$. Toute personne intéressée à poursuivre des études supérieures et à mener des recherches scientifiques en accord avec ses conditions était priée de remplir le formulaire de demande et de l'expédier au service du secrétariat de l'Office au 501, avenue Viger à Montréal ${ }^{42}$.

Si les membres de l'Office reconnaissaient la valeur fondamentale de la recherche scientifique et la nécessité absolue de former des chercheurs,

39. Circulaire de l'Office provincial des recherches scientifiques pour l'année 1938, Fonds de l'Institut de botanique (E118)A1, 1254, Archives de l'Université de Montréal.

40. Pour les bourses d'études du CNR, voir, Yves Gingras, Les origines..., op. cit., 87-101.

41. Circulaire de l'Office provincial des recherches scientifiques pour l'année 1938, Fonds de l'Institut de botanique (E118)A1, 1254, Archives de l'Université de Montréal.

42. Ibid. En 1945, le secrétariat déménage au 4, rue Notre-Dame Est puis, en 1954, au 1650, rue Berri, circulaires de l'Office provincial des recherches scientifiques pour l'année 1945 et 1954, Fonds de la faculté des sciences (E0096/C4, 0018), Archives de l’Université de Montréal. 
force est de constater que les universités francophones du Québec étaient très mal outillées pour mener à bien cette mission. Les programmes d'études supérieures de Laval et de Montréal visaient avant tout à «former des professeurs pour l'enseignement secondaire afin d'améliorer le niveau de l'enseignement des sciences dans les collèges, les séminaires et les couvents ${ }^{43}$ ». Il n'est donc pas étonnant qu'entre 1920 et 1937, seulement 19 diplômés des facultés de science du Québec obtiennent des bourses du CNR pour poursuivre leurs études aux cycles supérieurs ${ }^{44}$. Yves Gingras a montré comment les bourses du Conseil national de recherches, particulièrement les studentships et les fellowships, défavorisaient nettement les universités moins bien pourvues en équipement de laboratoire et dont les étudiants n'avaient pas la chance de participer à des projets de recherche dès le premier cycle ${ }^{45}$. C'était évidemment le cas des universités Laval et de Montréal, où la recherche est demeurée une activité marginale dans les départements et facultés de sciences jusqu'à la fin de la Seconde Guerre mondiale. Pour briser ce cercle vicieux, selon lequel il fallait avoir fait de la recherche pour pouvoir continuer à en faire, le CNR établit en 1919 les bursaries afin d'encourager ceux qui ont bien réussi au premier cycle à entreprendre des études supérieures ${ }^{46}$. Mais même en comptabilisant ce type de bourses, on voit que l'organisme fédéral n'a décerné aux universités Laval et de Montréal qu'environ 520 bourses d'études entre 1917 et $1958^{47}$. Au cours de la même période, l'Université McGill reçoit à elle seule plus de 1650 bourses d'études du CNR ${ }^{48}$. Outre le fait que ces données confirment la place secondaire qu'occupent les universités francophones dans le champ universitaire canadien de l'époque, elles montrent avant tout l'importance de l'Office dans le développement de la recherche au Québec (francophone).

La possibilité de financement que l'Office offrait aux étudiants intéressés à poursuivre leurs études à la maîtrise et au doctorat était importante pour assurer la reproduction de la communauté scientifique francophone naissante. De 1938 à 1960, l'Office a grandement contribué à la formation de nouveaux chercheurs québécois en octroyant 482 bourses d'études à des étudiants de $2^{\mathrm{e}}$ et $3^{\mathrm{e}}$ cycles des facultés de sciences du Québec. Comme le montre le graphique 1, ce sont les étudiants de l'Université de Montréal

43. Luc Chartrand et al., op. cit., 247.

44. Ibid., 239-249.

45. Yves Gingras, Les origines..., op. cit., 88-89.

46. Ibid.

47. National Research Council Review, 1958.

48. Ibid. 
qui ont reçu le plus de bourses (289), viennent ensuite les étudiants de l'Université Laval (121) et ceux de l’Université McGill (72). Parmi eux, 173 ont décroché une maîtrise et 56 un doctorat (graphique 2). Ces chiffres, qui peuvent paraître négligeables à l'égard de nos standards actuels, sont loin d'être banals. Mentionnons, à titre indicatif, que l'Université de Montréal et l'Université Laval ont décerné, entre 1936 et 1960, 746 diplômes d'études supérieures en sciences ${ }^{49}$. Si l'on additionne tous les «diplômés de l'Office», soit 229 , on se rend compte que l'agence gouvernementale a attribué des bourses d'études à près du tiers de ces diplômés. Par ailleurs, si nous ne prenons que les bourses données dans les universités francophones, au nombre de 410, on voit que l'Office accordait en moyenne 18 bourses d'études par année aux étudiants des universités francophones, alors que le CNR n'en accordait que 12. L'Office a donc distribué plus de bourses d'études aux étudiants francophones du Québec que le CNR, et ce, dans un laps de temps plus court et avec des budgets incommensurablement plus petits.

GRAPH I Q UE I

\section{Nombre de bourses d'études décernées annuellement par l'Office provincial des recherches scientifiques selon les universités québécoises 1938-1960}

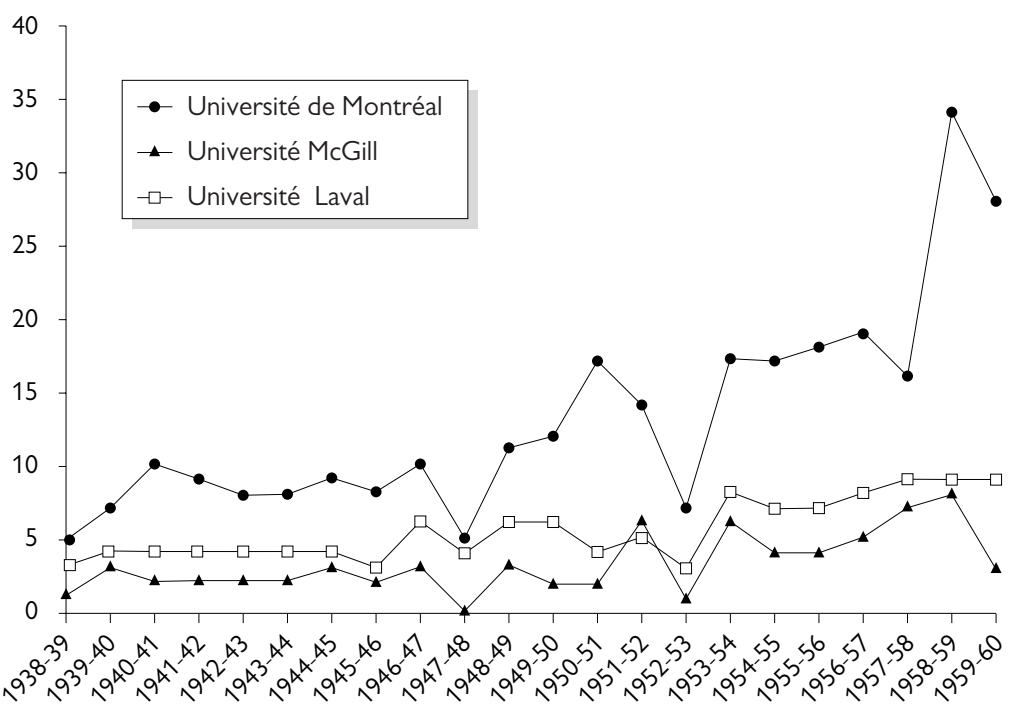

Source : Rapport général du Ministre des Affaires municipales, de l'Industrie et du Commerce de la province de Québec, 1942, 1943 et 1959. Office provincial des recherches scientifiques, Thèses et rapports des boursiers, Archives du ministère de l'Industrie, du Commerce et de la Technologie (EI6), 1960-0I-035/2I à 55, ANQ, Québec.

49. Raymond Duchesne, op. cit., 102. 


\section{GRAPH I Q U E 2}

\section{Nombre de maîtrises et de doctorats obtenus avec l'aide financière de l'Office provincial des recherches scientifiques 1938-1960}

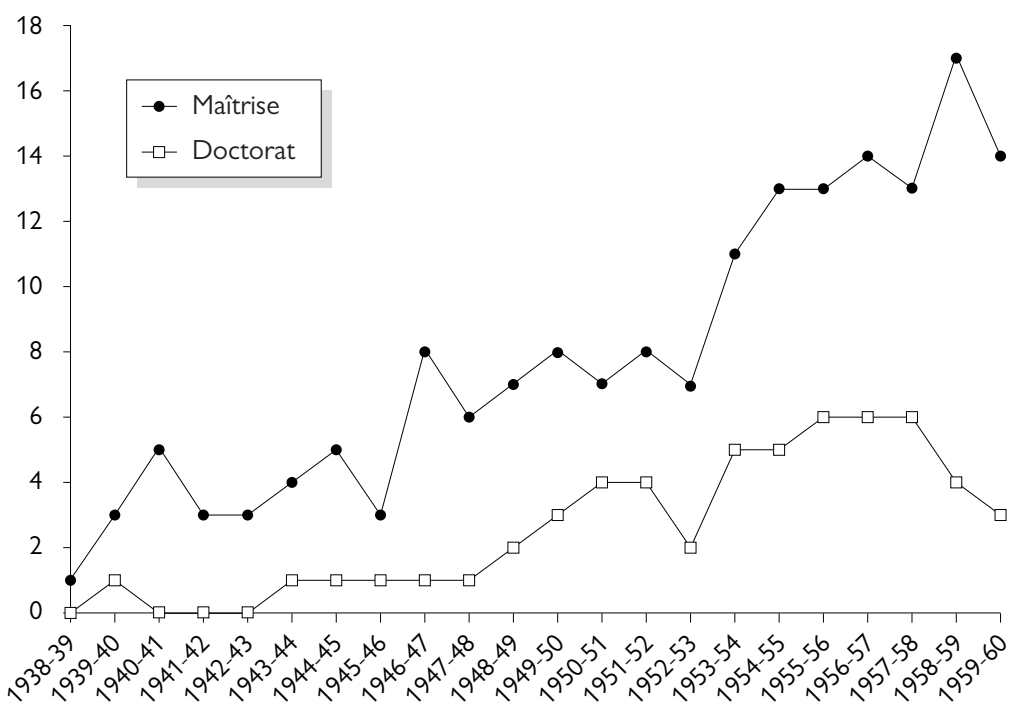

Source: Rapport général du Ministre des Affaires municipales, de l'Industrie et du Commerce de la province de Québec, 1942, 1943 et 1959. Office provincial des recherches scientifiques, Thèses et rapports des boursiers, Archives du ministère de l'Industrie, du Commerce et de la Technologie (EI6), I960-01-035/2I à 55, ANQ, Québec.

Il est donc certain que les bourses d'études supérieures offertes par l'Office ont permis à un plus grand nombre de jeunes Canadiens français d'obtenir des diplômes de $2^{\mathrm{e}}$ et de $3^{\mathrm{e}}$ cycles dans les universités de la province. Ces bourses ont également favorisé le développement de plusieurs disciplines, particulièrement la chimie et la physique, en assurant un certain renouvellement de leur corps disciplinaire. En effet, l'aide à la recherche, prodiguée pendant plus de 20 ans par l'Office, assurait un recrutement plus régulier d'étudiants des cycles supérieurs, là «où s'acquiert, en réalité, le métier de chercheur et la connaissance des règles du jeu» en vigueur dans chacune des disciplines scientifiques ${ }^{50}$. Parce que le discours sur l'importance de la recherche scientifique n'est pas suffisant pour assurer la formation et la reproduction d'une communauté scientifique nationale, nous croyons que l'Office a joué un rôle important dans l'institutionnalisation de la pratique scientifique au Québec en mettant à la disposition des jeunes chercheurs québécois des ressources matérielles essentielles à l'apprentissage de cette pratique. 


\section{LA RECHERCHE}

Comme nous l'avons vu, les fondateurs de l'Office entendaient non seulement former de nouveaux chercheurs par l'octroi de bourses d'études, mais ils visaient également à encourager les professeurs à s'investir davantage dans la recherche. Car si l'on souhaitait, par l'attribution de bourses d'études supérieures, faciliter l'initiation à la recherche, encore fallait-il que les professeurs soient en mesure, eux aussi, d'y consacrer un peu de leur temps. Contrairement à nos professeurs actuels, souvent accusés de négliger leur devoir d'enseignant pour dédier leur énergie à la recherche de subventions et à la course aux publications, leurs prédécesseurs de l'entre-deux-guerres s'adonnaient principalement à l'enseignement de premier cycle. À titre indicatif, mentionnons qu'entre 1918 et 1937, les professeurs de l'Université Laval ne reçoivent aucune subvention de recherche du CNR, alors que ceux de l'Université de Montréal reçoivent 15000 \$ et ceux de McGill autour de $50000 \$ \$^{51}$. Marie-Victorin obtient, à lui seul, $9650 \$$ entre 1926 et 1937 pour ses recherches ${ }^{52}$.

L'Office entendait donc accorder des subventions de recherche aux professeurs prêts à investir du temps et de l'énergie à l'étude de problèmes qui, dans une branche ou dans une autre de l'activité économique de la province, demandait une solution urgente. L'organisme fournissait les sujets de recherche, mais se disait ouvert à toute suggestion ${ }^{53}$. Ces bourses n'excédant pas 400 \$ étaient renouvelables à quiconque présentait la compétence scientifique jugée satisfaisante par l’Office. Ces subventions étaient attribuées selon les conditions suivantes : a) se mettre au travail dans les quinze jours qui suivent l'octroi officiel de la subvention; b) consacrer à la recherche un nombre d'heures fixé par entente entre le chercheur et l'Office; c) faire rapport de ses travaux tous les deux mois.

Le nombre de bénéficiaires de subventions de recherche a cependant diminué continuellement tout au long de l'histoire de l'Office. Bien qu'il nous ait été impossible de colliger les données relatives aux années 1950, cette assertion se vérifie aisément. En effet, l'augmentation draconienne des effectifs étudiants entraînait à l'Office une hausse substantielle des demandes de bourses. Les crédits additionnels obtenus à compter du début des années 1950 ont donc été absorbés, dans une large mesure, par cette augmentation de la demande en bourses d'études. De plus, la montée en 
flèche des inscriptions à l'université occasionnait, pour les professeurs, un alourdissement des charges d'enseignement et, par contrecoup, une limitation de leurs activités de recherche qui, on l'a vu, commençaient seulement à se développer.

C'est dans ce contexte que l'Office diminuait ses subventions de recherches aux chercheurs québécois. Il faut cependant noter que l'organisme subventionnaire opta alors pour des subvention allouées directement aux facultés de sciences tout en abaissant ses critères d'utilité, ce qui laissait la place au financement de recherches à caractère plus théorique ${ }^{54}$. Il faut dire aussi que les montants offerts par l'Office étaient de plus en plus négligeables comparés à ceux que les chercheurs pouvaient obtenir du $\mathrm{CNR}^{55}$.

La période la plus active au niveau de la recherche a sans aucun doute été celle qui s'étend de 1938 à 1948. Au cours de cette période, l’Office a financé des projets "d'intérêt national» comme les travaux d'Ivan $\mathrm{H}$. Crowell et de William Rowles, du Collège McDonald, sur les pathologies végétales observées dans les Cantons de l'Est durant les étés de 1937 et $1938^{56}$. L’année suivante, l'agence gouvernementale subventionnait le professeur Thomas M. Cameron, lui aussi du Collège McDonald, pour son enquête sur les parasites qui infectaient les achigans à petite bouche de la région d'Ottawa. À l'Université de Montréal, les recherches des professeurs Louis-Paul Dugual sur les huîtres, de Gaston Gosselin sur l'huile de foie de morue et de Roger Barré sur les miels de la province, sont autant d'exemples de recherches effectuées dans le cadre de l'inventaire des ressources naturelles. La priorité donnée à ce type de travaux n'a pourtant pas empêché l'Office de subventionner certaines recherches fondamentales, comme les travaux de HenriPaul Koenig, de l’Université Laval, sur les gerbes de mésotrons dans la radiation cosmique. Mais les recherches qui ont pris le plus d'envergure au

54. À partir de 1953-1954, l'Office attribua aux facultés de sciences de l’Université de Montréal et de l'Université Laval des montants de 2500 \$ qui devaient être alloués à la recherche. McGill, pour sa part, recevait $1500 \$$. Procès-verbal de la $31^{\mathrm{e}}$ réunion plénière de l'Office des recherches scientifiques, tenue au Château Frontenac, à Québec, le vendredi 9 mai 1952, Fond Georges Préfontaine (P0001), / A, 283, Archives de l'Université de Montréal. C'est aussi dans ce contexte que les travaux sur les émulsions photographiques du physicien Pierre Demers ont reçu un appui financier de l'Office.

55. National Research Council Review, 1950.

56. William Rowles, "A Study of Minor Element Deficiency Troubles in Crop Plants", McDonald College, 1938. Ivan H. Crowell, «Rusts of Oats, Timothy and Diseases of Clover and Photopathological Methods of Investigation ", McDonald College, 1938, Office provincial des recherches scientifiques, Thèses et rapports des boursiers, Archives du ministère de l'Industrie, du Commerce et de la Technologie (E16), 1960-01-035/21, ANQ, Québec. 
cours de la période sont celles qui ont porté sur Vicia Faba, communément appelée fève gourgane.

\section{LE « PROJET GOURGANE »}

Dès 1938, Jules Labarre, alors professeur de biochimie à l'Université de Montréal, est chargé, avec l'aide de ses étudiants, de mener des recherches sur les possibilités d'application industrielle de certaines propriétés de la fève. En 1941, près d'une dizaine de recherches, toutes dirigées par Labarre et subventionnées par l'Office, avaient été complétées ${ }^{57}$. Plusieurs de ces recherches ont fait l'objet de publications ${ }^{58}$. Les résultats étaient tellement encourageants que le président de l'Office, Paul Riou, était prêt à aller de l'avant et à procéder à la mise sur pied d'une installation semi-industrielle. Sa demande, auprès du ministre, d'un budget additionnel de $3000 \$$ pour mener à bien les essais préliminaires fut acceptée ${ }^{59}$. En 1943, il dépose un rapport au ministre sur l'état des travaux concernant le "projet gourgane». Déplorant l'attente excessive de certains instruments qui retarde la mise en marche de l'usine, Riou se montre néanmoins enthousiaste devant le progrès des recherches. Il écrit :

Par un procédé nouveau - dont le détail est contenu dans un brevet actuellement rédigé que nous déposerons à Washington et à Ottawa, aussitôt que les circonstances le permettront - nous avons réussi à préparer des protéines

57. Lucien Delcourt, «Recherches sur la fève gourgane», Université de Montréal, 1938-1939. Paul David, «Étude comparée des protides de la fève soya, gourgane et caséine au point de vue de l'industrie des peintures et des vernis», Université de Montréal, 1939-1941. Robert Dostert, «Étude sur la résinification des principaux constituant de la gourgane», Université de Montréal, 19391941. Lucien Delcourt, «Composition et propriétés générales des protides de la gourgane et fractionnement de ces protides», Université de Montréal, 1939. Roland Paquette, «Étude comparée des protides de la gourgane au point de vue de l'alimentation», Université de Montréal, 1940. Jules Labarre, «Protéines de la fève gourgane», Université de Montréal, 1940. Paul Hébert, «Protides de la gourgane dans l'industrie des colles», Université de Montréal, 1941. Paul Lapointe, «Étude comparée de la digestibilité in vitro. Par la pancréatine, de la farine de gourgane et de quelques autres farines», Université de Montréal, 1941. Roméo Poupart, «Influence de la température sur les transformations diastiques du lait de gourgane», Université de Montréal, 1941, Office provincial des recherches scientifiques, Thèses et rapports des boursiers, Archives du ministère de l'Industrie, du Commerce et de la Technologie (E16), 1960-01-035/21 à 55, ANQ, Québec.

58. Jules Labarre, Lucien Delcourt, «Les protides des semences de la fèves gourgane», Revue canadienne de biologie, 1,1 (1942) : 72-87. Jules Labarre, Robert Dostert, «Dénaturation progressive des protides des semences de gourganes", Revue canadienne de biologie, 1,5 (1942) : 504-522. Jules Labarre, Paul David, «Étude comparée sur quelques dérivés des protides totaux de la gourgane et de la caséine ", Revue canadienne de biologie, 1,1 (1943) : 72-86. Jules Labarre, Saul Pfeffer, "Études sur les enzymes de la fève gourgane pendant la germination ", Revue canadienne de biologie, 2,2 (1946) : 233-246.

59. Voir les Comptes publics de la province de Québec, 1943. 
solubles dans l'eau, qui par dessiccation à l'air donnent un film qui devient rapidement soluble. Ces protéines peuvent donc servir de base à une peinture à l'eau qui, lorsqu'elle sera sèche, pourra être lavable même avec un savon. Nous en avons préparé qui résiste parfaitement à l'action mécanique du frottage et des détergents. Notre peinture se composerait en somme de protéines, de pigments - blancs ou colorés - et d'eau. Pour les peintures d'intérieur, elle serait très convenable et coûterait trois à quatre fois moins cher que les peintures existantes. La grande économie réside dans le fait que le véhicule, l'huile, la térébenthine ou l'alcool est remplacé par de l'eau ${ }^{60}$.

Les recherches ont également donné des résultats attrayants en ce qui concerne l'utilisation des résidus de l'extraction des protéines. En effet, Riou se disait confiant de pouvoir préparer, avec ces résidus, un produit dont le goût et la composition se rapprochent des extraits de viande que l'on trouvait, et que l'on trouve encore, sur le marché sous les noms d'Oxo et de Bovril. Le nouveau procédé d'extraction des protéines donnait également une colle parfaitement utilisable pour l'encollage du papier et la fabrication de contre-plaqués ${ }^{61}$.

Dans son rapport, le président de l'Office signale également que la petite usine d'essai sera opérationnelle dans les jours qui suivent. Presque tout le matériel nécessaire à la fabrication des produits mentionnés est en place et un groupe de financiers s'est chargé de fournir 600 livres de gourgane pour l'année en cours et autant pour l'année suivante. Nous avons découvert que ces financiers avaient acheté, pour la somme d'un dollar, le droit et le privilège exclusif d'acquérir et d'exploiter le procédé d'extraction des protéines de la fève gourgane ${ }^{62}$. Dans le cas où le groupe décidait de se prévaloir de son privilège d'achat, il s'engageait à fournir, directement ou par l'intermédiaire d'une compagnie, un capital de 50000 \$ pour l'installation d'une usine. Il s'engageait également à payer une royauté aux inventeurs du procédé à raison de $6 \%$ des profits annuels jusqu'à concurrence de $100000 \$$. Dans le cas où les financiers décidaient de ne pas exercer leur privilège d'achat, les inventeurs du procédé devaient rembourser le montant total des dépenses encourues par le groupe, lequel montant ne devait pas excéder $8000 \$ \$^{63}$.

60. Rapport sur l'état actuel des recherches concernant la gourgane et ses possibilités d'exploitation industrielle, 1943, Archives du registraire du ministère de l'Industrie, du Commerce et de la Technologie (E16), 1960-01-035/181.

61. Ibid.

62. Convention notariée $\mathrm{n}^{\circ}$ 17008, daté du 11 mai 1942, entre Paul Riou et al. (Jules Labarre et Jean J. Lévesque) et Rodolph E. Joron, Archives du registraire du ministère de l'Industrie, du Commerce et de la Technologie (E16), 1960-01-035/181.

63. Ibid. 
Bien qu'il nous ait été impossible d'établir l'issue de ces alliances, signalons que la demande de brevet fut effectivement déposée à Washington en 1944 et le brevet accordé en $1947^{64}$. Malheureusement, aucun indice ne nous permet de croire que les inventeurs ont touché des redevances pour l'exploitation commerciale de leur procédé, mais les travaux sur Vicia Faba se sont poursuivis jusqu'en 1946-1947, ce qui témoigne de l'importance ou à tout le moins de l'intérêt pour ces recherches ${ }^{65}$. C'est que la gourgane est une proche parente du soja dont les protéines étaient elles aussi employées pour l'alimentation des animaux et la production de substances servant à l'encollage du papier. Le Québec importait des quantités importantes de cette plante alors que la gourgane, plus riche en matières protéiques, était cultivable dans toute la province. Faisant état de la situation lors d'un discours prononcé devant les membres de la société des conférences de l'Université d'Ottawa, le sous-ministre Louis Coderre jugeait que :

La culture de la gourgane et son emploi se traduiraient en revenus considérables pour nos gens. Libérés sur ce point de notre sujétion économique des ÉtatsUnis, il nous serait loisible, par la culture en grand de la gourgane, d'enrichir certaines populations actuellement assez pauvres de la région du Saguenay. Ainsi, une véritable révolution, favorisée par nos découvertes, s'opérerait dans l'économie du Nord de certains comtés peu propices aux cultures ordinaires ${ }^{66}$.

Chose certaine, cette révolution n'a pas eu lieu. À notre connaissance, il n’y a jamais eu de «culture en grand de la gourgane» au Saguenay, bien que l'on ait, depuis des générations, cultivé la plante pour préparer la fameuse soupe aux gourganes. Ce que cet épisode de l'histoire de l'Office montre,

64. Official Gazette of the United States Patent Office, 602 (septembre 1947) : 588, 2, 427, 760, "Process for Extraction of Selective Horse Bean Proteins in Acid Media», Frédéric A. Beique, Outremont, Québec, Canada, serial n ${ }^{\circ}$ 527, 678. Frédéric A. Beique était un étudiant de Jules Labarre associé au "projet gourgane». Étrangement, ses rapports de recherches ne figurent pas au fonds d'archives de l'Office. Tout ce dont nous disposons sur ce chercheur est contenu dans le procès-verbal de la $7^{\mathrm{e}}$ réunion plénière de l'Office des recherches scientifiques, tenue au nouveau palais de Justice à Montréal, le samedi 30 mars 1940, Fonds Georges Préfontaine (P0001), /A, 282, Archives de l'Université de Montréal. Il s'agit du montant de sa bourse (500\$).

65. Nous estimons que l'Office a investi tout près de $15000 \$$ dans ces travaux sur la gourgane. Germain J. Brisson, «Horse Bean as a Potential Source of Protein in Poultry Feeding», Université McGill, 1946-1947, Office provincial des recherches scientifiques, Thèses et rapports des boursiers, Archives du ministère de l'Industrie, du Commerce et de la Technologie (E16), 1960-01-035/29, ANQ, Québec.

66. Savoir où l'on va et comment y aller, conférence de Louis Coderre, sous-ministre des Affaires municipales, de l'Industrie et du Commerce, prononcée devant les membres de la Société des conférences de l'Université d'Ottawa, le 13 novembre 1938, Fonds Esdras Minville (P035), Archives des HEC. 
c'est qu'au niveau de la recherche, l'État provincial était inscrit dans une dynamique relationnelle avec l'université et l'industrie, dynamique dont la logique fondamentale consistait à commercialiser les produits de la recherche effectuée dans les laboratoires universitaires. D’ailleurs, l'exemple de la gourgane n'est pas unique. En 1938, l'Office commande au laboratoire d'électronique appliquée de l’École polytechnique de Montréal des recherches en vue de développer une méthode de séchage par hautes fréquences électriques afin de desservir l'industrie forestière et alimentaire ${ }^{67}$.

Au sein de ce triptyque - université, industrie, gouvernement - l'Office peut être vu comme l'une des premières structures institutionnelles de liaison entre l'université et l'entreprise privée au Québec. Il est donc clair que, pour l'État québécois, la recherche scientifique devait être orientée en fonction des besoins de l'entreprise privée. Que cette politique fût vouée à l'échec dans le contexte socio-économique du Québec d’avant 1960 ne change rien au fait que les polémiques actuelles sur le «nouveau mode de production des connaissances" prennent une tournure plutôt secondaire lorsqu' il s'agit du cas québécois. Bien que ce concept ait un intérêt heuristique certain en histoire des sciences en donnant des outils conceptuels permettant d'analyser les transformations survenues dans la pratique scientifique, l'analyse historique permet de montrer que certaines des tendances identifiées comme nouvelles par Michael Gibbons et ses collaborateurs pour l'après-guerre peuvent être observées dès la première moitié du $\mathrm{xx}^{\mathrm{e}}$ siècle ${ }^{68}$.

\section{VERS UN CENTRE DE RECHERCHES INDUSTRIELLES AU QUÉBEC}

Avec la fin de la Seconde Guerre mondiale et surtout au cours des années 1950, le discours sur l'importance d'un organisme provincial de recherche n'est plus l'apanage exclusif des hommes de science et devient même le cheval de bataille des industriels et entrepreneurs de la province qui réclament désormais l'intervention du gouvernement dans le domaine de la «recherche appliquée».

C'est que la conversion de l'industrie de guerre à une production de temps de paix s'est opérée sans difficultés majeures, grâce à la conjonction

67. Voir Jean-François Auger, «La commercialisation des produits de la recherche en génie du laboratoire d'électronique appliquée de l'École Polytechnique de Montréal, 1937-1975 », Histoire, économie et société, 20,1 (2001): 110.

68. Michael Gibbons et al., The New Production of Knowledge : the Dynamics of Science and Research in Contemporary Societies (Londres, Sage, 1994). Pour une revue claire et synthétique des critiques récentes qui ont été adressées aux modèles du «nouveau mode de production des connaissances» de Gibbons et de ses collègues, ainsi que du "triple hélice» de Henry Etzkowitz et Loet Lydesdorff, voir Terry Shinn, "Change or Mutation?...», op. cit. 
d'un certain nombre de facteurs, comme l'élaboration d'une politique de plein emploi par le gouvernement fédéral, la forte demande de rattrapage de la part des consommateurs, qui ont dû se priver pendant la crise et la guerre, et enfin, la reconstruction de l'Europe ${ }^{69}$. L'essor économique fulgurant de l'après-guerre, qui se poursuit sans heurts jusqu'à la récession de 1957, favorise la croissance de la production industrielle. Pour de nombreux économistes et représentants du monde des affaires, les sciences et les techniques sont au cœur de cette nouvelle croissance. Dans cette perspective, les progrès techniques et scientifiques deviennent condition générale de possibilités nouvelles : formes d'organisation du travail, apparition de nouveaux produits et de nouveaux processus de fabrication, etc. En d'autres termes, la science et les techniques sont, selon cette doctrine économique, l'essence même du progrès économique et social. Les contrats du gouvernement et les immenses ressources qu'il met au service des entreprises durant la guerre ont certainement contribué à changer la mentalité des milieux d'affaires qui veulent désormais continuer à bénéficier des avantages d'un État entrepreneur. La «conversion» des États occidentaux à l’ordre libéral keynésien ne laisse pas les hommes d'affaires du Québec indifférents et ils cherchent à obtenir leur part de cette nouvelle prospérité économique sans précédent ${ }^{70}$.

Il faut dire que les industriels ne sont pas les seuls à réclamer l'intervention du gouvernement au cours des années 1950. Sans vouloir entrer dans les détails de la Révolution tranquille, rappelons seulement que c'est fort d'un consensus social établi à la fin de cette décennie que le gouvernement du Québec peut faire de l'État le moteur et l'instrument privilégié d'un «rattrapage» et d'une «modernisation » considérés essentiels par tous. La mission économique de l'État québécois semblait inéluctable. Jean Lesage l'exprimait clairement peu après son arrivée au pouvoir : «Le seul puissant moyen que nous possédons, c’est l'État du Québec [...]. Si nous refusions de nous servir de notre État, par crainte ou préjugé, nous nous priverions alors de ce que qui est peut-être l'unique recours qui nous reste pour survivre comme minorité ${ }^{71}$.»

69. Paul-André Linteau, René Durocher, Jean-Claude Robert et François Ricard, Histoire du Québec contemporain, 2 : Le Québec depuis 1930 (Montréal, Boréal, 1986), 187-188.

70. Sur la "conversion» du Québec à l'ordre libéral keynésien, voir Paul-André Linteau et al., op. cit., 429-441.

71. Jean Lesage, le 3 juin 1961, cité par Pierre Fournier, Les sociétés d'État et les objectifs économiques du Québec : une évaluation préliminaire (Québec, Éditeur officiel, 1979), 15 p. 
Ainsi, en 1959, la Chambre de Commerce de la province de Québec dépose un mémoire au ministre de l'Industrie et du Commerce réclamant la création d'un centre de recherche gouvernemental semblable à ceux mis sur pied en Alberta et en Ontario. Pour les auteurs du mémoire, ce centre de recherche :

aurait pour mission la mise en valeur de nos ressources naturelles de façon à ce que la province en tire le plus grand profit possible, l'intensification de la production, l'amélioration de la qualité des produits permettant ainsi à notre industrie de faire face à la concurrence extérieure. Il fournirait les services de spécialistes dont la petite et la moyenne industrie ont besoin pour résoudre leurs problèmes, tant au moyen de conseils qu'au moyen de recherches expérimentales faites à la demande de telle ou telle industrie ${ }^{72}$.

Le mutisme des membres de la Chambre de Commerce sur l'existence de l'Office, patent dans cet extrait, se comprend aisément, surtout en cette fin des années 1950. C'est que leurs revendications à l'endroit du gouvernement portaient sur la mise sur pied de vastes laboratoires de "recherches appliquées", gérés et financés par l'État. Les activités scientifiques de l'Office et son apport à la vie économique, par trop liés à un régime qui s'écroulait, étaient sans doute considérés comme marginaux et dépassés, incompatibles avec le projet «interventionniste» d'un centre de recherche gouvernemental mis de l'avant par les représentants du monde des affaires $^{73}$. De toute façon, les autorités du ministère de l'Industrie et du Commerce (MIC), auquel était attaché l'Office, appuyaient les revendications de la Chambre de Commerce, non seulement parce qu'il y avait communauté d'intérêts entre les deux organismes, mais aussi parce que l'idée de planification économique et de développement industriel et technologique faisait de plus en plus consensus au sein du gouvernement provincial. De plus, les besoins des entreprises trop petites pour assumer les frais d'une installation de recherche permanente étaient de plus en plus criants $^{74}$.

72. Mémoire sur la recherche, Annexé au mémoire de la Chambre de Commerce de la Province de Québec à la Commission Parent, septembre 1962, 202-208.

73. Il serait impossible de savoir si les membres de la Chambre connaissaient l'existence de l'Office sans cette seule et unique allusion à l'organisme : «le Ministère de l'Industrie et du Commerce subventionne certaines recherches en accordant des octrois à des étudiants gradués". Mémoire sur la recherche, Annexé au mémoire de la Chambre de Commerce de la Province de Québec à la Commission Parent, septembre 1962, 203.

74. Le Mémoire du ministère de l'Industrie et du Commerce à la Commission Parent, 1962, est assez révélateur à ce sujet. 
Dans cette nouvelle conjoncture, l'Office apparaît comme une institution désuète, appartenant à une époque révolue, même si elle tente de s'adapter aux conditions changeantes de son milieu.

\section{UNE TENTATIVE DE RÉFORME}

Afin de le rendre plus conforme aux idées et impératifs du temps, plusieurs projets de réformes de l'Office ont été soumis au gouvernement au cours des années 1950. Un des plus chauds partisans de cette nouvelle orientation que l'on tente de donner à l'Office est sans aucun doute son nouveau président, Jules Labarre.

Né le 5 juillet 1904 à Yamachiche, Labarre obtient son baccalauréat en pharmacie de l'Université de Montréal en 1923. Deux ans plus tard, il devient titulaire d'une licence en chimie et, la même année, l'université le recommande au Secrétariat de la Province pour une «bourse d'Europe». Il part alors pour Paris et s'inscrit à l'Institut Pasteur où il se livre à des recherches sur la cellulose sous la direction de Gabriel Bertrand, chef de service de l'Institut et professeur à la faculté des sciences de l'Université de Paris $^{75}$. Après la soutenance de sa thèse en décembre 1927, il sillonne l'Europe pour finalement effectuer des études postdoctorales en chimie analytique à l'Université de Strasbourg. À nouveau française depuis 1918 et véritable fenêtre intellectuelle de la France sur l'Europe, l'Université de Strasbourg joue un rôle moteur dans la recherche universitaire effectuée au service des entreprises durant l'entre-deux-guerres ${ }^{76}$. Labarre complétait donc sa formation par un stage en "recherche appliquée». À son retour au Québec en 1929, le docteur Georges Baril le prend sous son aile et lui confie une charge de cours à la faculté des sciences de l'Université de Montréal $^{77}$. Il commence alors une longue carrière universitaire qui sera couronnée, en 1972, par le statut de professeur émérite. Reconnu pour ses talents d'organisateur, Labarre est membre fondateur de l'Association générale des diplômés de l'Université de Montréal (1934), il accepte, en 1952, de diriger l'organisation du laboratoire de biochimie de l'Hôpital Notre-Dame.

À son arrivée à la présidence de l'Office, en 1948, Labarre est chimisteconseil dans l'industrie pharmaceutique et l'auteur de nombreuses publi-

75. Le Canada, lundi 16 janvier 1928.

76. Françoise Olivier-Utard, Le mécénat industriel à la faculté des sciences de Strasbourg (19181998) : recherche libre ou prestation de service aux entreprises?, communication, Université de Toronto, mai 2002.

77. Entrevue avec Jules Labarre, le 13 novembre 1986, par Jacques Saint-Pierre, P137/fonds APRUDM/Entrevues d'histoire orale, Archives de l'Université de Montréal. 
cations scientifiques dont la Société royale du Canada a reconnu la valeur en le recevant parmi ses membres ${ }^{78}$. Le ministre de l'Industrie et du Commerce, Paul Beaulieu, compte sur son dynamisme pour redonner vigueur à l'agence gouvernementale. Lors d'un discours prononcé à Québec dans le cadre du congrès de l'ACFAS de 1948, Labarre affiche ses couleurs :

Il faut donc aider aux recherches scientifiques parce qu'elles contribuent à élever l'esprit humain en le mettant en face des inconnus physiques de la nature, inconnus qui lui sont accessibles et qu'il a pour mission de résoudre; parce qu'elles sont un facteur de civilisation; parce qu'elles conditionnent fortement le milieu économique et social dans lequel nous évoluons ${ }^{79}$...

Labarre se faisait donc le chantre d'une conception de la science qui trouvait de plus en plus d'écho au sein de la communauté scientifique et du monde des affaires, conception selon laquelle la recherche scientifique pouvait répondre aux nécessités et aux impératifs du développement industriel. Nous avons vu qu'à titre de directeur des recherches concernant le "projet gourgane", Labarre avait acquis une expérience certaine en la matière. Bien au fait du potentiel et des lacunes de l'Office, il va tenter de le transformer selon sa vision de la recherche scientifique.

Ainsi, dès le début des années 1950, Labarre persuade le ministre Beaulieu qu'il est temps que le gouvernement accorde plus d'importance aux travaux scientifiques à portée industrielle. Sans vouloir restreindre le travail à accomplir en ce qui a trait à la formation de nouveaux chercheurs, Labarre propose la création, au sein de l'Office, d'un comité des recherches appliquées ${ }^{80}$. Ce comité, formé par Labarre lui-même, Paul Riou, Donatien Marion, Louis Coderre et Fortunat Fortier, avait comme mandat de définir des projets de recherches sur le potentiel industriel des ressources naturelles, d'étudier l'utilisation possible des déchets industriels et d'aider, par tous les moyens possibles, à augmenter la productivité des entreprises qui en faisaient la demande. Pour mener à bien cette besogne, Labarre prônait la constitution d'un centre de documentation, la mise sur pied d'un labo-

78. Le Devoir, jeudi 8 janvier 1948.

79. Manuscrit de la causerie de Jules Labarre, président de l'Office provincial des recherches scientifiques, au Congrès annuel de l'ACFAS, Québec, 1948, Ministère de l'Industrie et du Commerce de la province de Québec, Archives du registraire du ministère de l'Industrie, du Commerce et de la Technologie (E16), 1960-01-035/183. L'italique est de nous.

80. Lettre de Jules Labarre à Paul Beaulieu, ministre, et Louis Coderre, sous-ministre de l'Industrie et du Commerce, daté du 27 mars 1951, Archives du registraire du ministère de l'Industrie, du Commerce et de la Technologie (E16), 1960-01-035/183. 
ratoire et l'embauche d'un "chimiste-enquêteur». Ce dernier était chargé de s'enquérir auprès des entreprises afin d'établir, par ordre de priorité, les sujets de recherche de haute valeur économique. Cette tâche a finalement été confiée à Germain McAvoy, diplômé en chimie de l'Université de Montréal possédant de nombreuses années d'expérience dans l'industrie ${ }^{81}$.

Profitant, en 1954, du déménagement de l'Office dans les nouveaux bureaux du MIC au Palais du Commerce à Montréal, Labarre met sur pied le centre de documentation de l'Office dont les rapports de recherche de ses boursiers formaient l'essentiel de la collection. Selon Fortunat Fortier, ce centre de documentation, dont pouvaient bénéficier les industriels qui avaient à résoudre des problèmes techniques, comprenait également «beaucoup d'ouvrages scientifiques, de traités, d'annuaires, de formulaires, de manuels touchant des questions très variées tant au point de vue de la science pure que des applications pratiques ${ }^{82} »$. Quant au laboratoire, il fut constitué à grands frais, puisque dès 1953 Labarre évalue à $10452 \$$ le montant total des dépenses encourues pour les instruments qui le composent ${ }^{83}$. D’abord aménagé dans le laboratoire personnel du professeur de biochimie à l'Université de Montréal, il fut ensuite transporté dans les nouveaux locaux de l'Office. Notons, par ailleurs, que les cinq autres organismes provinciaux de recherche de l'époque mettent également sur pied des équipes de recherche et des laboratoires auxquels peuvent faire appel les entreprises trop petites pour assumer les frais d'une installation de recherche permanente ${ }^{84}$.

C’est que tout au long des années 1950, les demandes de renseignements et de consultation de la part des entrepreneurs augmentent constamment ${ }^{85}$.

81. Lettre de recommandation de Jules Labarre adressée au sous-ministre Louis Coderre, daté du 23 avril 1951, Archives du registraire du ministère de l'Industrie, du Commerce et de la Technologie (E16), 1960-01-035/183.

82. Office des recherches scientifiques. Revue générale de ses travaux depuis sa fondation, 1954, Archives du registraire du ministère de l'Industrie, du Commerce et de la Technologie (E16), 1960-01-035/180.

83. Lettre de Jules Labarre à Louis Coderre, daté du 23 juin 1953, Archives du registraire du ministère de l'Industrie, du Commerce et de la Technologie (E16), 1960-01-035/83.

84. Frances Anderson, Olga Berseneff-Ferry et Paul Dufour, «Le développement des conseils de recherche provinciaux : quelques problématiques historiographiques», Scientia canadiensis, 7,1 (1983) : 31. Il s'agit du Research Council of Alberta, du Ontario Research Fondation, du Saskatchewan Research Council, du British Columbia Research Council et du Nova Scotia Research Council.

85. Il est tout de même intéressant de souligner que dès ses premières années de fonctionnement, l'Office répond à des demandes de renseignements concernant des procédés de fabrication et des recettes en tout genre, autant pour le gouvernement que pour les particuliers. À titre 
Bien qu'il n'existe, à notre connaissance, que très peu d'études spécialisées sur la croissance de la petite et moyenne entreprise (PME) québécoise au cours des années 1950, de nombreux indices nous laissent croire qu'elle a connu un développement rapide au cours de cette période. D’abord, le nombre d'établissements manufacturiers est passé de 11670 en 1950 à 12112 en 1956, soit une augmentation moyenne de 73 établissements par année ${ }^{86}$. Il est certain que la plupart de ces nouvelles entreprises étaient des $\mathrm{PME}^{87}$. Ensuite, le revenu net des entreprises individuelles non agricoles s'est accru de $88 \%$ entre 1950 et $1963^{88}$. D'ailleurs, si l'on prend le secteur manufacturier dans son ensemble, on voit que la valeur de la production a presque doublé, alors que les investissements, privés et publics (les dépenses en réparation et entretien étant exclues), sont multipliés par deux entre 1950 et $1960^{89}$.

Cette croissance des PME et du secteur manufacturier durant les années 1950 est nettement perceptible dans l'augmentation des demandes de consultation technique et scientifique adressées au laboratoire de l'Office. En 1951, par exemple, l'Office effectue un travail d'expertise pour l'entreprise Gérald Élie, de Drummondville, ainsi qu’une mise au point pour l'entreprise Wooden Creamery de Weedon ${ }^{90}$. En 1954, l'agence provinciale prête ses services à la Dolbo Inc. qui désire obtenir des renseignements sur

d'exemple, citons le cas d'un entrepreneur de Marieville qui, en 1940, avait l'intention d'établir une petite industrie pour la fabrication de dentifrice et qui désirait recevoir des informations à ce sujet. Lettre de François De Simonne à Paul Riou, daté du 20 novembre 1940, Archives du registraire du ministère de l'Industrie, du Commerce et de la Technologie (E16), 1960-01-035/83. En 1944, l'agence gouvernementale agit même en véritable conseil scientifique lorsque le ministre Beaulieu charge ses membres de lui remettre un rapport sur les problèmes à prévoir pour l'aprèsguerre. Après une revue exhaustive des problèmes possibles, l'Office recommandait, sans surprise, la création de grands travaux publics dans le secteur des richesses naturelles. Conservation des ressources naturelles, mémoire présenté par l'Office provincial des recherches scientifiques, document de 8 pages, Fonds de l'Institut de botanique (E118) A1, 1254, Archives de l'Université de Montréal. En 1949, le ministre récidive et commande une recherche sur l'utilisation des déchets du tabac. Louis Trochu, «L'utilisation des déchets du tabac», Université de Montréal, 1949. Office provincial des recherches scientifiques, Thèses et rapports des boursiers, Archives du ministère de l'Industrie, du Commerce et de la Technologie (E16), 1960-01-035/29, ANQ, Québec.

86. Paul-André Linteau et al., op. cit., $2: 238$.

87. Yves Bélanger, Pierre Fournier, L'entreprise québécoise. Développement historique et dynamique contemporaine (Montréal, Hurtubise HMH, 1987), 60.

88. Marcel Daneau, «Évolution économique du Québec, 1950-1965 », L'Actualité économique (janvier 1966) : 659-692.

89. Ibid., 688.

90. Lettre de Jules Labarre à Louis Coderre, datée du 6 novembre 1951, Archives du registraire du ministère de l'Industrie, du Commerce et de la Technologie (E16), 1960-01-035/183. 
le marché des carbonates dans les domaines pharmaceutique et médical ${ }^{91}$. En 1959, le laboratoire de l'Office effectuait des travaux sur les moyens d'extraction du sulfure de molybdène à partir du catalyseur Simon (industrie des lubrifiants), des expériences sur une méthode de dosage de l'arsenic dans le bismuth et conduisait de nombreux essais d'extraction des impuretés dans le métal brut ${ }^{92}$.

Afin de faire face à ces demandes, l'Office n'avait eu d'autres choix que d'accroître ses moyens. À l'instar de l'ensemble de l'appareil étatique québécois, l'Office prend une expansion considérable sans toutefois modifier ses prémisses non interventionnistes ${ }^{93}$. C'est exactement cette orientation, donnée à l'Office comme à toutes les agences étatiques du gouvernement, qui sera remise en question lors de l'arrivée au pouvoir des libéraux de Jean Lesage.

Dès 1961, «l'équipe du tonnerre» crée, au sein du ministère de l'Industrie et du Commerce, le Conseil d'orientation économique afin de planifier le développement économique de la province. Ce conseil avait été constitué à partir de l'ancien Bureau des recherches économiques auquel on avait intégré l'Office en 1960. Le détail des refontes institutionnelles dans lesquelles l'Office a été impliqué prend un aspect plutôt secondaire dans le nouveau rapport de force qui s'établit au tournant des années 1960 et qui mène à sa disparition. Nous n'en ferons donc pas un traitement exhaustif. Car il faut bien admettre que la réaffectation de son secrétaire, Fortunat Fortier, de son technicien en laboratoire, Germain McAvoy, et la réorganisation de son laboratoire, ne nous permettent pas de comprendre les causes de son démembrement. Ce qu'il faut retenir ici c'est le rôle important que va jouer le Conseil d'orientation économique dans le projet d'un organisme provincial de recherche. Ce nouveau conseil avait, comme la plupart des institutions créées au début des années 1960, l'objectif lointain de moderniser la structure industrielle et les équipements d'infrastructures et, surtout, de donner aux francophones une plus large part du contrôle de l'économie québécoise.

91. Dans un rapport à Louis Coderre sur les travaux du laboratoire pour l'année 1953-1954, Fortunat Fortier mentionne 15 sujets de recherches qui ont fait l'objet de travaux au laboratoire de l'Office cette année-là. Les expériences ont porté autant sur les différentes méthodes de fonte du verre que sur les possibilités de fabriquer du tribromure d'or, Archives du registraire du ministère de l'Industrie, du Commerce et de la Technologie (E16), 1960-01-035/181.

92. Selon le Rapport du Ministre de l'Industrie et du Commerce de la province de Québec, 1959. Malheureusement, le rapport ne fait aucune mention des entreprises pour lesquelles ces recherches ont été conduites.

93. Voir James Iain Gow, Histoire de l'administration publique québécoise 1867-1970 (Montréal, Presses de l’Université de Montréal, 1986), 269-270. 
Il est important, pour les besoins de l'analyse, de mentionner que le nationalisme économique est une caractéristique quasi constante des réformes entreprises par ceux qui voulaient être "maîtres chez nous». En 1970, Jacques Parizeau l'exprimait sans ambiguïté :

Au Québec, il faut faire intervenir l'État. C'est inévitable. C'est ce qui nous donne une allure plus à gauche. Si nous avions, au Québec, 25 entreprises Bombardier et si nous avions des banques très importantes, la situation serait peut-être différente. Nous n'avons pas de grosses institutions, il faut donc les créer ${ }^{94}$.

Pour espérer "créer 25 entreprises Bombardier», il fallait mettre sur pied un centre de recherche au service des entreprises et surtout au service des petites et moyennes entreprises québécoises, afin qu'elles prennent de l'expansion. Bien qu'il ne soit pas question ici de montrer en détail les événements qui ont mené à la création du Centre de recherches industrielles du Québec (CRIQ), détails qui ont été présentés avec précision par Raymond Duchesne, il suffit de signaler que l'État-providence en formation s'étend non seulement à l'éducation et au bien-être social, mais également aux entreprises. Comme nous l'avons vu avec l'épisode du "projet gourgane », l'État du Québec a depuis longtemps tenté de mettre la recherche scientifique au service de l'industrie. Dans les années 1960, il se donne les moyens de ses ambitions. Les nouveaux rapports qui prennent alors forme au sein des relations gouvernement/université/industrie sont dominés par l'État qui acquiert le monopole du financement de la recherche, tant universitaire que para-universitaire.

Les liens entre l'université et l'industrie en sont conséquemment réduits à leur plus simple expression. Il faut cependant noter que les universitaires avaient énormément souffert de la crise de financement des institutions d'enseignement supérieur qui avait cours depuis 1945. Leurs préoccupations principales allaient donc tout naturellement vers le financement des universités et leur souci d'établir la supériorité de la "recherche fondamentale» sur la «recherche appliquée» en est un aboutissement logique ${ }^{95}$. Même que certains membres de l'Office ont refusé de cautionner l'élargissement de ses cadres et de ses fonctions, alors que la question du financement universitaire

94. Cité dans Philip Resnick, «La vengeance des huguenots : sur l’héritage de la Révolution tranquille ", dans Robert Comeau, dir., Jean Lesage et l'éveil d'une nation (Sillery, Presses de l’Université du Québec, 1989), 325.

95. Voir Cri d'alarme... La civilisation scientifique et les Canadiens français (Sainte-Foy, Les Presses de l’Université Laval, 1963), 142 p. 
demeurait en suspens ${ }^{96}$. Il y a donc là un autre pan de l'explication des échecs qui entourent le projet de réforme de l'Office au cours des années 1950.

Tout se passe comme si la mise en place de l'État-providence et sa prise en charge du financement de la recherche scientifique plaçaient en incubation les relations université/industrie. La création du CRIQ abonde en ce sens puisqu'il s'agit d'un instrument de recherche mis à la disposition du secteur privé et presque totalement indépendant du monde universitaire. De plus, on le voit bien aujourd'hui, les relations entre le secteur économique et les universités ne se sont vraiment renouées qu'à partir du début des années 1980, décennie marquée par les coupures budgétaires draconiennes des gouvernements en matière de programmes sociaux, d'éducation et de recherche. Mais il s'agit là de considérations qui dépassent largement les limites de notre enquête. Elles pourraient faire à elles seules l'objet d'une étude complète.

\section{CONCLUSION}

L'Office provincial des recherches scientifiques ne fut pas un «échec», ni même un "échec relatif» dans le développement de la science au Québec. L’agence gouvernementale créée par Esdras Minville a consacré toute son existence à la formation d'une main-d'œuvre scientifique, main-d'œuvre encore au stade embryonnaire au moment où l'Office distribuait ses premières bourses d'études à la fin des années 1930. Bien sûr, l'émergence de chercheurs francophones au Québec fut attribuable à la fondation de la Faculté des sciences de l'Université de Montréal et à celle de l'École supérieure de chimie de l'Université Laval. Mais si pour la première fois des Canadiens français pouvaient entreprendre une carrière scientifique sans avoir à étudier à McGill ou à quitter la province, très peu d'entre eux poursuivaient leurs études aux cycles supérieurs. Le CNR fait d'ailleurs savoir dès 1920 aux dirigeants de l'Université de Montréal qu'aucun Canadien français n'a obtenu de bourse de son programme, en vigueur depuis 1917. En 1937, année de la création de l'Office, seulement une vingtaine de chercheurs francophones avaient bénéficié d'une aide financière du $\mathrm{CNR}^{97}$. Rappelons que l'Office a distribué quelque 480 bourses d'études à des étudiants diplômés des facultés de sciences du Québec entre 1938 et 1960. En l’absence de structures institutionnelles comme l'Office, il est donc illusoire de croire que les scientifiques canadiens-français auraient pu participer aux activités 
constitutives de leurs disciplines et assurer le renouvellement de leur communauté en tant que telle. Évidemment, l'Office n'apparaît pas dans le vide et n'est pas le primus mobile de tout le développement scientifique du Québec avant 1960. Mais il peut être évoqué, au même titre que l'on évoquait jusqu'ici le CNR, la loi des «bourses d'Europe» ou même la Fondation Rockefeller, comme une étape importante dans l'institutionnalisation de la pratique scientifique au Québec. Le lecteur pourrait objecter, non sans raison, qu'il faudrait une analyse détaillée de la trajectoire professionnelle des boursiers de l'Office, afin d'établir la proportion d'entre eux qui ont entrepris une carrière scientifique, que ce soit en milieu universitaire, industriel ou gouvernemental. Cet exercice aurait le mérite de jeter un meilleur éclairage sur la spécificité de la contribution de l'Office par rapport aux organismes mentionnés plus haut. Quoi qu’il en soit, la présente recherche se voulait une première ébauche, un premier relevé «topographique» d'un terrain historique laissé en friche par les historiens des sciences au Québec. D'autres travaux pourront aborder cet aspect du devenir des boursiers.

Finalement, notre enquête a révélé que pour l’État du Québec, la recherche scientifique devait être liée aux besoins et aux impératifs du développement économique. Les différents moyens qu'il s'est donné pour atteindre cet objectif ont énormément varié selon les conjonctures idéologiques, socio-économiques et scientifiques. Entre 1938 et 1953, l'Office subventionne des projets de recherches «d'intérêt national» visant, pour la plupart, à résoudre des problèmes liés à l'exploitation des ressources naturelles de la province. Avec l'épisode du "projet gourgane», nous avons vu comment l'agence gouvernementale cherchait à orienter les produits de la recherche universitaire vers des applications pratiques à l'industrie formant ainsi un véritable organe de liaison entre les laboratoires universitaires et l'entreprise privée. Au cours des années 1950, la demande grandissante des entrepreneurs en consultation technique et scientifique force l'Office à se doter d'un comité des recherches appliquées, d'un laboratoire et d'un centre de documentation, services qui apporteront une aide non négligeable aux PME florissantes de l'époque.

Si aujourd'hui il est parfois difficile de distinguer la recherche académique de la prestation de services aux entreprises, il ne faudrait pas conclure trop rapidement à un phénomène nouveau et sans précédent dans l'histoire des sciences. Le cas du Québec montre des liens par moment très étroits entre les universités, l'État et l'entreprise privée, liens qui rendent peu pertinente la distinction conventionnelle entre recherche fondamentale et recherche appliquée, et ce, bien avant le triomphe du néolibéralisme. 